\title{
Câncer de Esôfago: Perfil das Manifestações Clínicas, Histologia, Localização e Comportamento Metastático em Pacientes Submetidos a Tratamento Oncológico em um Centro de Referência em Minas Gerais
}

\author{
Esophageal Cancer: Profile of Clinical Manifestations, Histology, Location and
} Metastatic Behavior in Patients Undergoing Cancer Treatment at a Cancer Center in Minas Gerais

\author{
Nonato Mendonça Lott Monteiro', Daniel Francisco de Araúioº ${ }^{2}$ Eric Bassetti-Soares ${ }^{3}$, José Pedro Ferreira de Bastos Vieira ${ }^{4}$, \\ Marcos Rivânio Marinho dos Santos ${ }^{5}$, Pedro Paulo Lopes de Oliveira Júnior ${ }^{4}$, Tatiana Siqueira Jacques Delgado
}

\section{Resumo}

O câncer de esôfago (CE) é uma neoplasia com uma incidência crescente, com taxas de mortalidade próximas às taxas de incidência. Sua etiologia está associada ao tipo histológico da doença, sendo o carcinoma de células escamosas o mais comum e fortemente relacionado ao tabagismo e etilismo, e o adenocarcinoma associado ao esôfago de Barrett. Além desses fatores sabidamente conhecidos, o risco de desenvolver este tumor está aumentado em pessoas que ingerem alimentos e bebidas quentes (mate) e que possuem nutrição deficiente (hipovitaminose $\mathrm{A}, \mathrm{C}$ e E); há também uma predisposição genética que ainda é pouco definida. Manifestaçōes clínicas comuns durante a evolução dessa doença incluem: disfagia, odinofagia, desconforto retroesternal, hiporexia, náusea, vômitos, emagrecimento. Tais queixas merecem uma avaliação criteriosa, pois, quando essas se manifestam, a doença já se encontra, na maioria das vezes, em um estágio avançado, não sendo possível uma abordagem curativa destes pacientes. Os protocolos de tratamento do CE incluem cirurgia, quimioterapia e radioterapia, mas o melhor tratamento ainda é motivo de estudos. Este trabalho teve como objetivo descrever e analisar o perfil das manifestações clínicas, a relação entre o tipo histológico e a localização, idade e comportamento metastático e terapêutica, de pacientes portadores de CE submetidos a tratamento oncológico no Centro de Oncologia e Radioisótopos (COR), Ipatinga-MG. Foi feita análise retrospectiva dos prontuários de 109 pacientes com diagnóstico de CE, de maio de 2004 a fevereiro de 2007. Para cálculos estatísticos, foi utilizado o programa Epi Info 6.04d.

Palavras-chave: Neoplasias esofágicas; Metástase neoplásica; Histologia

${ }^{1}$ Estudante do $12^{\circ}$ período da Faculdade de Medicina do Vale do Aço e estagiário do departamento de Radioterapia do Centro de Oncologia e Radioisótopos de Ipatinga (MG), Brasil

${ }^{2}$ Estudante do $12^{\circ}$ período da Faculdade de Medicina da UNIPAC/Barbacena (MG), Brasil

${ }^{3}$ Médico, Doutor em Gastroenterologista, Professor e Coordenador de Pesquisa da Faculdade de Medicina do Vale do Aço, Ipatinga (MG), Brasil

${ }^{4}$ Médico Radioterapeuta do Centro de Oncologia e Radioisótopos de Ipatinga (MG), Brasil

${ }^{5}$ Estudante do $8^{\circ}$ período do curso de Biomedicina da UNIPAC/Ipatinga (MG), Brasil

Trabalho realizado: Centro de Oncologia e Radioisótopos de Ipatinga (MG), Brasil - Faculdade de Medicina do Vale do Aço

Endereço para correspondência: Nonato Mendonça Lott Monteiro. Rua Nilo Peçanha, nº 270 - Areal - Ipatinga (MG), Brasil - CEP: $35160-271$.

E-mail: nonatomlm@yahoo.com.br 


\section{INTRODUÇÃO}

O câncer de esôfago (CE) é uma neoplasia altamente agressiva, sendo considerada a sexta causa de morte por câncer no mundo. Apresenta uma alta taxa de incidência em países, como: China, Japão, Cingapura e Porto Rico. No Brasil, consta entre os dez mais incidentes e, para o ano de 2008, são esperados cerca de 10.550 casos novos de câncer de esôfago, sendo 7.900 em homens e 2.650 em mulheres. A maioria dos casos ocorrerá nas regiōes sul e sudeste: 8.400 casos novos. No ano de 2000 , foi o sexto tipo de maior letalidade, com 5.037 óbitos $^{1}$.

A etiologia do CE envolve uma interação de diversos fatores de risco (FR), como: idade, história familiar e associação genética, além de muitos fatores extrínsecos ${ }^{2,3}$. Entre estes, estão a ingestão de álcool, tabagismo, uso de nitrosaminas e aflotoxinas, infecçōes locais por fungos, deficiência de Riboflavina e vitamina A (ingesta baixa de frutas e legumes) e ingesta excessiva de erva mate $^{2,4}$. Algumas afecções como o megaesôfago, estenoses cáusticas do esôfago e esôfago de Barrett têm suas importantes contribuições na etiologia do $\mathrm{CE}^{5,6,7}$. Alguns fatores ocupacionais como exposição em longo prazo à poeira de sílica, hidrocarbonetos aromáticos policíclicos e metais também têm sido estudados como FR para o este tipo de câncer ${ }^{8,9}$.

O tipo escamocelular ou carcinoma de células escamosas (CCE) é o mais frequente, entretanto, estudos em países ocidentais evidenciam um declínio modesto na frequência desse tumor, e uma elevação dramática na frequência do tipo adenocarcinoma $(\mathrm{AC})^{10}$.

$\mathrm{O}$ diagnóstico do $\mathrm{CE}$ frequentemente é tardio uma vez que o principal sintoma, disfagia, não ocorre até que o tumor tenha crescido o suficiente para causar sintomas obstrutivos ${ }^{11}$. Os pacientes se ajustam à sua maior dificuldade de deglutição, alterando progressivamente sua dieta de alimentos sólidos para líquidos. Com a progressão da obstrução, dor e salivação excessivas ocorrem habitualmente, juntas com perda ponderal progressiva, sangramento, dor torácica e vômitos ${ }^{2,12,13}$.

Este estudo objetivou descrever e analisar o perfil das manifestações clínicas e avaliar a relação entre o tipo histológico e a localização, idade e comportamento metastático do $\mathrm{CE}$, em pacientes que foram submetidos a tratamento oncológico no Centro de Oncologia e Radioisótopos (COR) da cidade de Ipatinga, Minas Gerais, referência para o tratamento dessa patologia na região.

\section{MATERIAL E MÉTODOS}

Foi realizado um estudo longitudinal, descritivo e retrospectivo de 109 prontuários de pacientes com diagnóstico de CE, atendidos no COR, de maio de 2004 a fevereiro de 2007. Através da revisão dos prontuários, foram avaliadas as seguintes variáveis: idade, gênero, local da moradia (urbana ou rural), hábitos de vida (se etilista e/ou tabagista), história e tipo de câncer na família, data do diagnóstico após o aparecimento do primeiro sintoma, data do início do tratamento após o diagnóstico, manifestações clínicas, localização da doença, padrão histológico da doença, tratamento realizado, duração do tratamento, se houve recidiva ou persistência da doença e se o tratamento foi custeado pela medicina privada ou pública.

Dados categóricos estão representados por valores absolutos e percentagens, enquanto valores quantitativos contínuos, por média e desvio-padrão. $\mathrm{Na}$ comparação das variáveis categóricas, utilizou-se o teste do Quiquadrado ou teste exato de Fisher, quando aplicável, e, nas variáveis contínuas, o teste $t$ de Student. Para a análise dos dados, empregou-se o programa Epi-info 6.04d (CDC, 2001). O valor de $\mathrm{p}=0,05$ foi considerado estatisticamente significante.

A condução ética da pesquisa foi pautada pela Resolução 196/96, do Conselho Nacional de Saúde, e de acordo com a declaração de Helsinque.

\section{RESULTADOS}

No período analisado, a amostra de CE correspondeu a 4,8\% (109/2264) dos pacientes atendidos no COR, destes, $74,3 \%$ eram do gênero masculino (81/109). A idade variou de 39 a 88 anos $(62,9 \pm 11,8$ anos), sendo superior entre as mulheres $(69,2$ versus 60,7 anos; $p=0,0009)$. A Tabela 1 resume os dados relativos às faixas etárias ao diagnóstico e mostra que a de 51 a 60 anos representa quase um terço $(29,9 \%)$ dos pacientes avaliados. A Tabela 2 mostra o número e a frequência de algumas variáveis estudadas.

A história familiar de neoplasias estava presente em $37,1 \%$ desses pacientes (36/97), sendo mais frequente

Tabela 1. Distribuição da frequência da faixa etária ao diagnóstico e do gênero de pacientes com CE

\begin{tabular}{c|c|c}
\hline $\begin{array}{c}\text { Faixa efária ao } \\
\text { diagnóstico }\end{array}$ & $\mathbf{N}(\%)$ & $\begin{array}{c}\text { Masculino/ } \\
\text { Feminino }\end{array}$ \\
\hline 31 até 40 anos & $2 / 107(1,9)$ & $2 / 0$ \\
\hline 41 até 50 anos & $16 / 107(14,9)$ & $16 / 0$ \\
\hline 51 até 60 anos & $32 / 107(29,9)$ & $26 / 6$ \\
\hline 61 até 70 anos & $26 / 107(24,3)$ & $16 / 10$ \\
\hline 71 até 80 anos & $23 / 107(21,5)$ & $16 / 7$ \\
\hline 81 até 90 anos & $8 / 107(7,5)$ & $3 / 5$ \\
\hline
\end{tabular}


Tabela 2. Distribuição da frequência de algumas variáveis socioambientais e sua distribuição por gênero de pacientes com CE

\begin{tabular}{l|c|c}
\multicolumn{1}{c|}{ Variáveis } & N (\%) & Homem/Mulher \\
\hline Etilismo & $78 / 108(72,2)$ & $65 / 13$ \\
\hline Tabagismo & $64 / 108(59,3)$ & $58 / 06$ \\
\hline História familiar de câncer & $36 / 98(36,7)$ & $26 / 10$ \\
\hline Moradores de zona urbana & $99 / 109(90,8)$ & $72 / 27$ \\
\hline Moradores de zona rural & $10 / 109(9,2)$ & $9 / 1$ \\
\hline Tratamento custeado pela Medicina Pública & $102 / 109(93,6)$ & $74 / 28$ \\
\hline Tratamento custeado pela Medicina Privada & $7 / 109(6,4)$ & $7 / 0$ \\
\hline
\end{tabular}

a história familiar de câncer em esôfago, estômago e laringe (Gráfico 1).

$\mathrm{O}$ primeiro sintoma mais frequente foi a disfagia, relatada por $76,9 \%$ dos pacientes $(83 / 108)$, seguida por dor retroesternal $(17 / 108 ; 15,7 \%)$ e odinofagia $(3 / 108$; $2,8 \%)$. A perda de peso foi relatada por $104 / 108$ pacientes após o aparecimento do primeiro sintoma, sendo entre 10 e $15 \mathrm{~kg} \mathrm{em} \mathrm{41/108} \mathrm{(37,9 \% ).} \mathrm{Durante} \mathrm{a}$ evolução da doença, todos os pacientes apresentaram disfagia e odinofagia, além de $90,7 \%$ que apresentaram dor retroesternal (Gráfico 2).

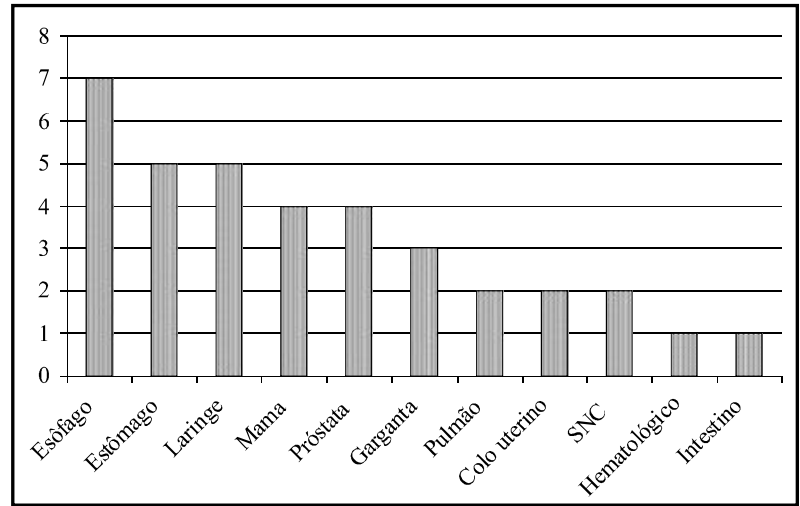

Gráfico 1. História familiar de neoplasias em pacientes com CE $(\mathrm{n}=97)$

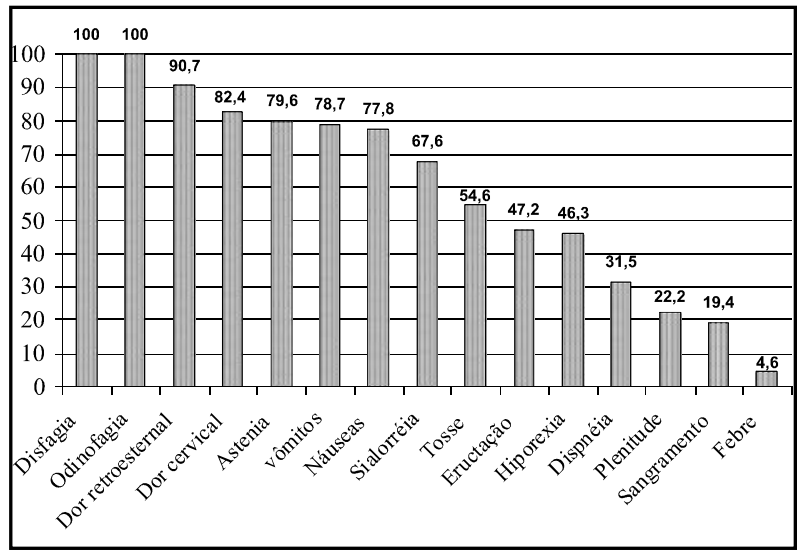

Gráfico 2. Manifestações clínicas apresentadas pelos pacientes durante o tratamento, em percentual $(n=108)$
Quanto ao tipo histológico feito através de Endoscopia Digestiva Alta com Biópsia, foram encontrados 11/109 casos (10,1\%) de AC e 98/109 $(89,9 \%)$ de CCE. O CCE acometeu pacientes mais jovens do que o $\mathrm{AC}(62,1$ versus 72,2 anos, $\mathrm{p}=0,008)$. $\mathrm{O}$ AC manifestou-se preferencialmente em esôfago inferior, enquanto o CCE comprometeu os três terços do esôfago indistintamente, sendo um pouco mais frequente no esôfago médio ( $\mathrm{p}=0,00008$ ) (Gráfico 3).

Não se observou associação significativa entre a idade e a presença de metástase no grupo de CCE. Entretanto, no grupo de pacientes com $\mathrm{AC}$, os pacientes que apresentavam tumor metastático eram estatisticamente mais jovens do que os sem metástases $(58,5$ versus 76,6 anos; $\mathrm{p}=0,02)$. Na comparação da idade com o comprometimento ganglionar, não foram observadas associaçōes estatisticamente significantes entre as variáveis, entretanto no grupo AC a média da idade em pacientes com disseminação linfática foi inferior em aproximadamente 12 anos $(67,0$ versus 80,6 anos; $\mathrm{p}=0,08)$.

Em 58,6\% dos pacientes (61/104), o diagnóstico foi confirmado após seis meses do aparecimento do primeiro sintoma (Gráfico 4) e, após o diagnóstico, em $90,8 \%$ dos pacientes (99/109) o tratamento foi instituído em até 30 dias.

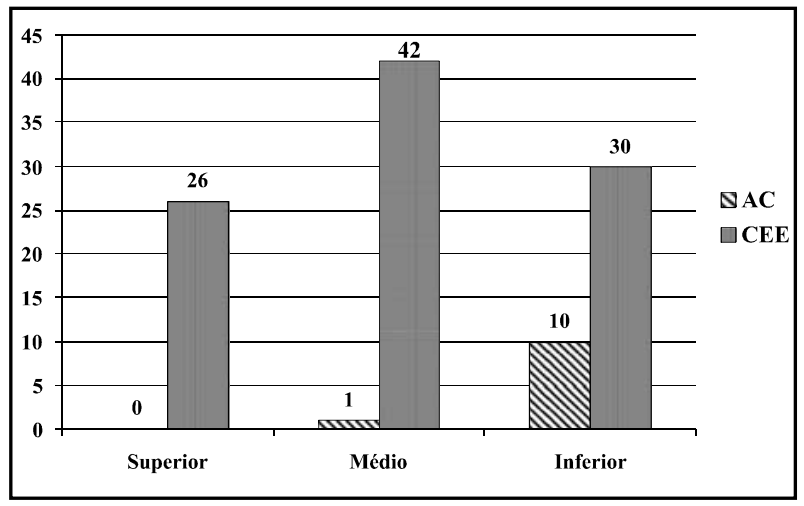

Gráfico 3. CE - Tipo histológico e local de acometimento ( $\mathrm{n}=$ pacientes) 


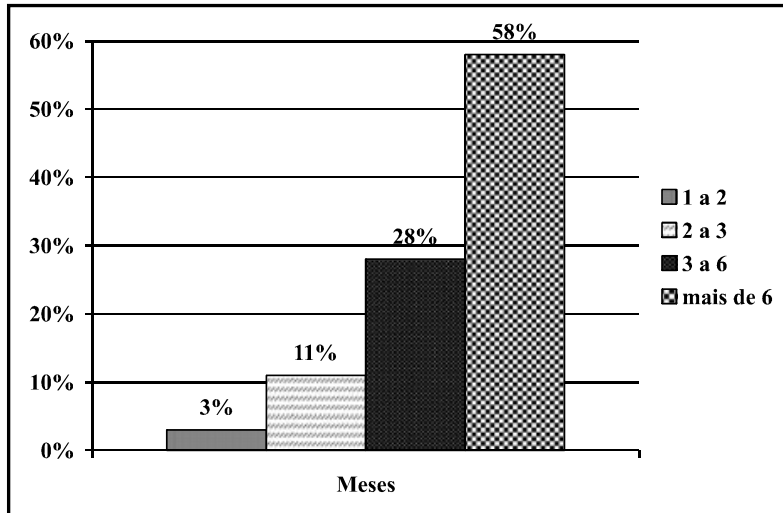

Gráfico 4. Intervalo (meses) entre o surgimento do primeiro sintoma e o diagnóstico de CE $(n=104)$

O tratamento radioterápico foi realizado em $98,1 \%$ dos pacientes avaliados. Aproximadamente, um terço $(38,3 \%)$ foi submetido à quimioterapia e apenas $1,9 \%$ à cirurgia. Dos pacientes acompanhados após o tratamento, que permaneceram em controle clínico no COR, 92,4\% (85/92) relataram melhora clínica, com remissão completa ou parcial de suas queixas. A persistência do tumor foi observada em $26 / 57$ (45,6\%) dos pacientes tratados e a recidiva em $6 / 55$ (10,9\%).

\section{DISCUSSÃO}

Existem poucos trabalhos científicos publicados no Brasil sobre CE. Estudos sobre os FR deste tipo de câncer já foram realizados no Rio Grande do Sul e em países como Uruguai, Argentina ${ }^{4}$, China ${ }^{14}$, Lituânia ${ }^{15}$, Taiwan ${ }^{16}$, Suécia ${ }^{17}$, entre outros.

No estudo atual, a amostra foi de 109 pacientes diagnosticados com $\mathrm{CE}$ e que foram encaminhados ao COR para receberem tratamento oncológico.

$\mathrm{Na}$ análise dos resultados, foi observado que o CE foi predominante em pacientes com idade superior aos 51 anos de idade e do gênero masculino, o que concorda com o que é descrito na literatura ${ }^{1,11}$.

Apesar de não alcançar nível de significância estatística, foram altas as porcentagens dos pacientes que relataram hábitos de etilismo e tabagismo $(72,2 \%$ e $59,3 \%$, respectivamente), o que já é relatado pela literatura, e sabidamente comprovado como fatores que aumentam a incidência deste tipo de tumor ${ }^{1,4,18}$. Também foi alta a porcentagem dos pacientes que relataram presença de familiar com história de câncer na família $(37,1 \%)$, o que nos leva a pensar e valorizar a etiologia genética e característica familiar desta doença na gênese do CE, conforme alguns estudos da literatura ${ }^{19,20}$.

Neste estudo, o CE foi predominante em moradores da zona urbana $(90,8 \%)$, o que também é compatível com informaçōes disponíveis na literatura que revelam alta incidência deste tipo de malignidade em pacientes moradores de áreas industrializadas e menor taxa de incidência em pacientes habitantes de área rural ${ }^{21}$.

A disfagia e a odinofagia foram as manifestações clínicas mais frequentes em nosso estudo (apresentadas por $100 \%$ dos 108 pacientes), o que reforça o curso clínico da doença, no qual o esôfago, não dispondo de uma barreira serosa, consequentemente, leva os seus tumores a crescerem em largura circunferencialmente até começarem a obstruir a passagem dos alimentos, tornando os pacientes incapazes de deglutir alimentos sólidos e, eventualmente, líquidos ${ }^{22}$.

Embora na literatura haja relatos de um aumento na frequência do tipo histológico $\mathrm{AC}$ em relação ao $\mathrm{CCE}^{10}$, em nosso estudo este segundo tipo foi o mais frequente $(89,9 \%$ versus $10,1 \%)$, distribuindo-se regularmente em todas as regiōes do esôfago, diferentemente do tipo AC que apresentou uma preferência pelo terço inferior (90,9\%), o que provavelmente é justificado pela sua etiologia que se relaciona ao esôfago de Barrett ou como uma extensão de um adenocarcinoma gástrico em região da cárdia gástrica ${ }^{23,24}$.

O diagnóstico tardio, maior do que seis meses na maioria dos pacientes $(58,6 \%)$, chama atenção para uma situação que provavelmente interferiu neste resultado: curso clínico insidioso da doença que se manifesta com disfagia quando o tumor já adquiriu tamanho o suficiente para causar obstrução, levando o paciente a alterar sua dieta para alimentos mais líquidos e ir se adaptando à evolução/crescimento do tumor com a alteração no padrão alimentar ${ }^{2,25}$.

Neste estudo, foi baixa a porcentagem de pacientes com história de tratamento cirúrgico $(1,9 \%)$, entretanto a quase totalidade dos pacientes foi submetida à radioterapia $(98,1 \%)$ e, uma parcela menor, à quimioterapia $(38,3 \%)$. A eficácia dos tratamentos instituídos foi avaliada pela melhora clínica. Durante o tratamento, elevado percentual de pacientes referiu melhora dos sintomas $(92,4 \%)$. Embora não tenha conseguido valor estatisticamente significante, foram altas as porcentagens de pacientes que evoluíram com persistência e/ou recidiva da doença após a realização da rádio e quimioterapia $(45,6 \%$ e $10,9 \%$, respectivamente). Nenhum dos três tratamentos, isoladamente, mostrou-se eficaz, e associação de duas ou até mesmo das três modalidades de tratamento devem ser avaliadas ${ }^{11}$. O melhor tratamento do CE ainda é motivo de pesquisas e discussōes, já que é um tumor com altas taxas de morbimortalidade ${ }^{22}$. 


\section{CONCLUSÃO}

O paciente com CE que procurou o COR no período estudado foi, na maioria das vezes, homem, a partir da sexta década de vida, que teve o diagnóstico confirmado há mais de seis meses após o aparecimento do primeiro sintoma. O CCE foi o tumor de esôfago predominante em nosso estudo e acometeu mulheres em idades mais avançadas do que os homens. O AC manifestou-se exclusivamente em pacientes com mais de cinquenta anos, mais tardiamente do que o CCE. Também houve associação importante entre tipo histológico e a localização no esôfago. Manifestaçôes clínicas frequentes durante a evolução da doença foram: disfagia, odinofagia e dor retroesternal, além de importante perda de peso. Durante o tratamento, a quase totalidade dos pacientes desfrutou de melhora de suas queixas, mas a doença persistiu e/ou recidivou em uma grande porcentagem, o que reforçou o comportamento altamente agressivo dessa neoplasia.

O CE trata-se de um tumor altamente agressivo e os pacientes com este tipo de neoplasia apresentam um prognóstico altamente reservado. Assim, uma investigação detalhada deverá ser feita com pacientes provenientes de áreas de alta incidência, que apresentem fatores de risco e/ou manifestaçōes clínicas associados a esta doença, para que os possíveis casos de CE tenham o diagnóstico estabelecido precocemente e as melhores medidas terapêuticas possam ser avaliadas e utilizadas no tratamento.

\section{Declaração de conflito de interesses: nada a declarar.}

\section{REFERÊNCIAS}

1- Instituto Nacional de Câncer. Estimativa 2008: incidência de câncer no Brasil [monografia na Internet]. Rio de Janeiro (Brasil): Instituto Nacional de Câncer; 2007 [citado em 2008 Jun 19]. Disponível em: http://www.inca.gov.br/ estimativa/2008/.

2- Dani R, Silva E, Nogueira CED. Gastroenterologia essencial. 3a ed. Rio de Janeiro: Guanabara Koogan; 2006.

3- Robertson EV, Jankowski JA. Genetics of gastroesophageal cancer: paradigms, paradoxes, and prognostic utility. Am J Gastroenterol. 2008;103(2):443-9. Epub 2007 Oct 9.

4- Dietz J, Pardo SH, Furtado CD, Harzheim E, Furtado AD. Fatores de risco relacionados ao câncer de esôfago no Rio Grande do Sul. Rev Assoc Med Bras. 1998;44(4)269-72.

5- Henry MA, Lerco MM, Oliveira WK. Esophageal cancer in patient with chagasic megaesophagus. Arq Gastroenterol. 2007;44(2):151-5.
6- Manoel-Caetano FS, Borim AA, Caetano A, Cury PM, Silva AE. Cytogenetic alterations in chagasic achalasia compared to esophageal carcinoma. Cancer Genet Cytogenet. 2004;149(1):17-22.

7- Cook MB, Wild CP, Everett SM, Hardie LJ, Bani-Hani KE, Martin IG, et al. Risk of mortality and cancer incidence in Barrett's esophagus. Cancer Epidemiol Biomarkers Prev. 2007;16(10):2090-6. Epub 2007 Sep 21.

8- Kamangar F, Strickland PT, Pourshams A, Malekzadeh R, Boffetta P, Roth MJ, et al. High exposure to polycyclic aromatic hydrocarbons may contribute to high risk of esophageal cancer in northeastern Iran. Anticancer Res. 2005;25(1B):425-8.

9- Wernli KJ, Fitzgibbons ED, Ray RM, Gao DL, Li W, Seixas NS, et al. Occupational risk factors for esophageal and stomach cancers among female textile workers in Shanghai, China. Am J Epidemiol. 2006;163(8):717-25. Epub 2006 Feb 8. Comment in: Am J Epidemiol. 2006;164(4):402; author reply 402-3. Am J Epidemiol. 2007;165(2):233-4.

10-Lagergren J. Increased incidence of adenocarcinoma of the esophagus and cardia. Reflux and obesity are strong and independent risk factors according to the SECC study. Lakartidningen. 2000;97(16):1950-3.

11-Queiroga RC, Pernambuco AP. Câncer de esôfago: epidemiologia, diagnóstico e tratamento. Rev Bras Cancerol 2006;52(2):173-8.

12-Siewert JR. Increasing dysphagia. Carcinoma: yes or no? MMW Fortschr Med. 2006;148(49-50):19-20.

13-Javle M, Ailawadhi S, Yang GY, Nwogu CE, Schiff MD, Nava HR. Palliation of malignant dysphagia in esophageal cancer: a literature-based review. J Support Oncol. 2006;4(8):365-73, 379. Comment in: J Support Oncol. 2006;4(8):377. J Support Oncol. 2006;4(8):378-9.

14-Li HQ, Diao YT, Li H, Zhou YZ, Yang YF, Fang XQ, et al. The risk factors related to esophageal squamous cell cancer in Feicheng county, China. Zhonghua Yu Fang Yi Xue Za Zhi. 2007;41 Suppl:S56-61.

15-Strumylaite L, Zickute J, Dudzevicius J, Dregval L. Saltpreserved foods and risk of gastric cancer. Medicina (Kaunas). 2006;42(2):164-70.

16- Wu IC, Lu CY, Kuo FC, Tsai SM, Lee KW, Kuo WR, et al. Interaction between cigarette, alcohol and betel nut use on esophageal cancer risk in Taiwan. Eur J Clin Invest. 2006;36(4):236-41.

17-Bahmanyar S, Ye W. Dietary patterns and risk of squamouscell carcinoma and adenocarcinoma of the esophagus and adenocarcinoma of the gastric cardia: a population-based case-control study in Sweden. Nutr Cancer. 2006;54(2):171-8.

18- Wu M, Zhao JK, Hu XS, Wang PH, Qin Y, Lu YC, et al. Association of smoking, alcohol drinking and dietary factors with esophageal cancer in high- and low-risk areas of Jiangsu Province, China. World J Gastroenterol. 2006;12(11):1686-93. 
19- Jain M, Kumar S, Upadhyay R, Lal P, Tiwari A, Ghoshal UC, et al. Influence of Apoptosis (BCL2, FAS), Cell Cycle (CCND1) and growth factor (EGF, EGFR) genetic polymorphisms on survival outcome: an exploratory study in squamous cell esophageal cancer. Cancer Biol Ther. 2007;6(10):1553-8. Epub 2007 Jul 7.

20-Sudo T, Mimori K, Nagahara H, Utsunomiya T, Fujita H, Tanaka Y, et al. Identification of EGFR mutations in esophageal cancer. Eur J Surg Oncol. 2007;33(1):44-8.

21- Gosschalk A, Carozza S. Cancer in rural areas: a literature review. Rural Healthy People 2010: a companion document to Healthy People 2010. College Station: The Texas A\&M University System Health Science Center, School of Rural Public Health, Southwest Rural Health Research Center; 2003. vol. 2.
22- Layke JC, Lopez PP. Esophageal Cancer: a review and update. Am Fam Physician. 2006;73(12):2187-94.

23- Koop H. Strategies for monitoring patients with Barrett's esophagus-contra. Dtsch Med Wochenschr. 2007;132(3435):1769. Comment on: Dtsch Med Wochenschr. 2007;132(34-35):1768.

24-Tu CH, Lee CT, Perng DS, Chang CC, Hsu CH, Lee YC. Esophageal adenocarcinoma arising from Barrett's epithelium in Taiwan. J Formos Med Assoc. 2007;106(8):664-8.

25- Weissfeld JL. Cyclin D1 and Esophageal Adenocarcinoma Risk: how good does a marker have to be? J Natl Cancer Inst. 2000;92(16):1282-3. Comment on: J Natl Cancer Inst. 2000;92(16):1316-21.

\section{Abstract}

Esophageal cancer (EC) is a type of tumor with an increasing incidence, and mortality rates close to incidence rates. Its etiology is associated with the histological type of the disease, with the squamous cell carcinoma as the most common and strongly related to smoking and alcoholic habits, as well as the adenocarcinoma associated with Barrett's esophagus. In addition to these well-known factors, the risk of developing this cancer is increased in people who ingest food and hot drinks (mate) and who have poor nutrition (hipovitaminose A, C and E); also, there is a genetic predisposition that is still poorly defined. Clinical manifestations common during the evolution of this disease include dysphagia, odynophagia, retroesternal discomfort, hiporexia, nausea, vomiting, weight loss. Such complaints deserve careful evaluation because when revealed, the disease is already, in most cases, in an advanced stage and a curative approach on these patients is not possible. Protocols for the EC treatment include surgery, chemotherapy and radiotherapy, but the best treatment is still cause for studies. This study aims to describe and analyze the profile of clinical manifestations, the relationship between the histological type and the location, age and metastatic behavior and therapy of patients with EC undergoing cancer treatment at the Oncology and Radioisotopes Center (COR), Ipatinga-MG. Retrospective analysis of records of 109 patients diagnosed with EC was made, from May 2004 to February 2007. For statistical calculations the program Epi Info 6.04d was used.

Key words: Esophageal Neoplasms, Neoplasm metastasis; Histology 